\title{
Presencia de Bordetella holmesii en brote epidémico de coqueluche en Chile
}

\author{
Carolina Miranda, Aniela Wozniak, Claudia Castillo, Enrique Geoffroy, Cecilia Zumarán, \\ Lorena Porte, Juan C. Román, Marcela Potin y Patricia García
}

\section{Presence of Bordetella holmesii in an outbreak of pertussis in Chile}

The incidence of whooping cough in Chile ranges from 4.1 and 7.5 per hundred thousand inhabitants. B. pertussis detection is performed by Real Time PCR (Q-PCR) directed to the insertion sequence IS481. However, this sequence is also found in the genome of $B$. bronchiseptica and $B$. holmesii. The latter is also a respiratory pathogen whose clinical features are similar to B. pertussis. However, it is important to differentiate between these species because in immunosuppressed patients $B$. holmesii is more likely to cause bacteremia and is less susceptible to erythromycin. The goal of this work is to measure prospectively and retrospectively the presence of $B$. holmesii in samples reported positive for B. pertussis in the period 2010-2011. During this period, 1994 nasopharyngeal specimens entered the laboratory for Bordetella sp. PCR, of which 224 were positive. The analysis by Q-PCR directed to the recA gene of $B$. holmesii of all 224 positive samples determined a prevalence of $B$. holmesii of $0.6 \%(12 / 1994)$. Because of its more aggressive behavior in immunosupressed patients and its different resistance pattern, routine screening of $B$. pertussis and B. holmesii is currently performed for all samples in which Bordetella sp PCR is initially detected.

Key words: Bordetella holmesii; insertion sequence IS481; polymerase chain reaction; diagnosis.

Palabras clave: Bordetella holmesii; secuencia de inserción IS481; reacción de la polimerasa en cadena; diagnóstico.

\section{Introducción}

$B$ ordetella pertussis es un patógeno respiratorio causante de coqueluche. Esta es una enfermedad infecto-contagiosa que se manifiesta por un cuadro clínico de tos de más de siete días, acompañada de paroxismos de tos, estridor inspiratorio o vómito inducido por la tos. En lactantes bajo 6 meses la infección puede cursar además con apneas repetidas. La transmisión es por contacto directo persona a persona, por medio de gotitas de secreción respiratoria y es altamente contagiosa. Esta bacteria es capaz de colonizar las vías respiratorias debido a la afinidad de sus factores de adherencia con el epitelio respiratorio ciliado ${ }^{1}$.

Las técnicas de laboratorio para el diagnóstico de $B$. pertussis son: el cultivo bacteriano en agar Regan Lowe, la inmunofluorescencia directa (IFD) y la reacción de polimerasa en cadena (RPC). El cultivo tiene una especificidad de $100 \%$ y una sensibilidad de $35-40 \%{ }^{2}$. La IFD tiene una sensibilidad variable que oscila entre 29 y $71 \%$; con respecto a la especificidad de esta técnica, se han descrito falsos positivos con B. bronchiseptica, Haemophilus influenzae y difteroides ${ }^{3}$. La RPC es la técnica más sensible durante las primeras etapas de la infección y es la recomendación actual para el diagnóstico según la
Academia Americana de Pediatría ${ }^{4}$. La RPC dirigida a la secuencia repetida IS481 exclusiva de Bordetella sp. es la más ampliamente usada ${ }^{5}$. El diagnóstico de Bordetella sp. en el Laboratorio de Microbiología de la Pontificia Universidad Católica de Chile (PUC) se realiza por RPC en tiempo real (Q-RPC) dirigida a esta secuencia. Durante la validación de esta técnica se determinó una correlación entre el ciclo umbral de amplificación (CT) y el número de bacterias presentes en la muestra. En base a la misma, se han fijado los siguientes puntos de corte: cuando el valor de CT es menor a 35 la muestra se considera positiva y cuando el valor es mayor que 40 la muestra se considera negativa, mientras que para valores entre 35 y 40 la muestra se considera indeterminada, lo cual corresponde a una baja carga bacteriana ${ }^{6}$. El genoma de $B$. pertussis tiene entre 80-100 copias de la secuencia IS481, razón por la cual constituyen un buen sitio blanco ya que aumenta la sensibilidad del ensayo ${ }^{7-8}$. Sin embargo, la RPC basada en la secuencia repetida IS481 puede dar falsos positivos con B. holmesii y con B. bronchiseptica debido a que la secuencia de inserción IS 481 se encuentra también en el genoma de estas especies aunque en menor número de copias (8-10 copias/genoma $)^{9-10}$. Se ha descrito que la secuenciación del gen $r e c A$ permite diferenciar entre $B$. pertussis y $B$. holmesii ${ }^{11}$. Bordetella holmesii
Pontificia Universidad Católica de Chile.

Escuela de Medicina.

Departamento de Laboratorios

Clínicos.

Laboratorio de Microbiología.

Proyecto financiado por Fondos Departamentales Concursables del Departamento de Laboratorios Clínicos. Pontificia Universidad Católica de Chile Los autores declaran que no tienen conflictos de interés.

Recibido: 22 de octubre de 2012 Aceptado: 16 de abril de 2013

Correspondencia a: Patricia García Cañete pgarcia@med.puc.cl 
fue descrita por primera vez como una nueva especie del género Bordetella en el año $1995^{12}$. Se la ha encontrado asociada a septicemia, endocarditis y falla respiratoria en pacientes inmunocomprometidos, especialmente en pacientes asplénicos e hiposplénicos ${ }^{11,13-14}$. Bordetella holmesii es también un patógeno respiratorio que produce un cuadro clínico similar a $B$. pertussis (tos prolongada), a pesar que carece de la toxina pertussis, responsable de la gravedad y letalidad en la infección causada por $B$. pertussis. Bordetella holmesii se aísla a partir de muestras de hisopado y aspirado nasofaríngeo en pacientes con clínica sospechosa de $B$. pertussis ${ }^{15}$; es detectada tanto en niños como en adultos ${ }^{15-16}$. Bordetella pertussis es la especie que con mayor frecuencia se asocia con cuadros clínicos respiratorios. Con menor frecuencia se encuentra $B$. parapertussis, y finalmente $B$. holmesii. Con respecto a $B$. bronchiseptica, está usualmente asociada a animales y ocasionalmente se aísla en pacientes inmunocomprometidos ${ }^{17}$. Si bien, cuando un examen de Q-RPC dirigido a la secuencia IS481 es positivo, se informa como $B$. pertussis, es importante diferenciar entre estas especies no sólo desde el punto de vista epidemiológico para conocer la incidencia de este patógeno respiratorio, sino también porque se han observado diferencias en la susceptibilidad a antimicrobianos. Ensayos in vitro de determinación de concentración inhibitoria mínima (CIM) mostraron que eritromicina es menos activa contra $B$. holmesii ${ }^{18}$.

$\mathrm{Si}$ bien la coqueluche en Chile es una enfermedad endémica con incidencias que varía entre 4,1 y 7,5 por cien mil habitantes, se han descrito brotes esporádicos, el último de ellos reportado entre los años 1997 y $2000^{19}$. A partir de septiembre del año 2010 se registró un aumento de los casos de Bordetella sp. notificados al Ministerio de Salud de Chile en relación a años anteriores, elevando la tasa de incidencia a 15,1 por cien mil habitantes en el año 2011 y fue definido como brote de la enfermedad por el MINSAL ${ }^{19}$. Concordantemente, en el Laboratorio de Microbiología de la PUC también se observó un aumento en el número de muestras recibidas para el análisis de Bordetella sp, así como también del número de casos positivos por Q-RPC basada en la secuencia IS481. Junto

Tabla 1. Secuencia de partidores y sondas utilizados para la detección de

B. pertussis y B. holmesii

$\begin{array}{llc}\text { Especie } & \text { Secuencia } & \text { Ref. } \\ \text { B. pertussis } & \text { IS481F: 5' - ATCAAGCACCGCTTTACCC-3' } & 7 \\ & \text { IS481R: 5' -TTGGGAGTTCTGGTAGGTGTG-3' } & \\ & \text { Sonda IS481TQ: 5' -6FAM-AATGGCAAGGCCGAACGCTTCA-BHQ-1-3' } & \\ \text { B. holmesii } & \text { BHrecA F: 5' -CGGTTCGCTGGGTCTCG-3' } & 10 \\ & \text { BHrecA R: 5' -CCCGCGGCAGACCAC-3' } & \\ & \text { Sonda BHrecA: 5' -6FAM-CATCGCATTGGGCG-MGBNFQ-3' }\end{array}$

con el aumento de casos positivos, se observó un aumento en el número de casos indeterminados (CT entre 35 y 40), que podría ser explicado por un aumento en la incidencia de la infección por $B$. holmesii dado que tiene un menor número de copias de la secuencia IS481, y por lo tanto mayores valores de $\mathrm{CT}^{10}$. El objetivo de este trabajo es determinar prospectiva y retrospectivamente la presencia de $B$. holmesii en muestras informadas como positivas para B. pertussis en el período 2010-2011, y además determinar si el aumento del número de resultados indeterminados durante el brote de $B$. pertussis corresponde a un aumento de la incidencia de $B$. holmesii.

\section{Material y Método}

Muestras. Se analizaron 1.994 muestras provenientes de pacientes ambulatorios y hospitalizados, en los cuales se solicitó la detección de B. pertussis por Q-RPC durante el período 2010-2011. El 47\% (934/1.994) eran pacientes de sexo masculino con un promedio de edad de 13,2 años (rango: 0-64 años) y una mediana de 8 años. En el caso de pacientes de sexo femenino (n: 1.060), el promedio de edad fue de 15,5 años (rango: 0-66 años) con una mediana de 11 años. Las muestras correspondieron a hisopado o aspirado nasofaríngeo y una vez recibidas en el Laboratorio de Microbiología UC, se realizó la extracción del ADN por un método comercial QIAamp® ADN Mini kit (QIAgen, Hilden, Alemania) según las instrucciones del fabricante. Los ADNs se almacenaron a $-20^{\circ} \mathrm{C}$.

Detección de Bordetella sp. por Q-RPC. Se realizó para todas las muestras una Q-RPC con sonda Taqman dirigida a la secuencia de inserción IS48 $1^{8}$ (Tabla 1). A las muestras positivas para la secuencia de inserción IS481, al día siguiente se les realizó además una Q-RPC con sonda Taqman dirigida al gen recA específica para $B$. holmesii ${ }^{11}$ (Tabla 1). Las muestras recibidas desde enero de 2010 hasta junio de 2011 fueron analizadas retrospectivamente para la presencia de $B$. holmesii y las recibidas a partir de julio de 2011 fueron analizadas en forma prospectiva. Por lo tanto, $48 \%$ de las muestras fueron analizadas retrospectivamente y el resto en forma prospectiva. Las que resultaron negativas para el gen $r e c A$, se informaron positivas para $B$. pertussis.

Las muestras se analizaron en un equipo StepOne Real-Time PCR System (Applied Biosystems). La mezcla de reacción en ambos casos tenía un volumen final de 20 $\mu \mathrm{L}$ y contenían $1 \mathrm{X}$ Taqman ${ }^{\circledR}$ Fast Universal PCR Master Mix (2x), Sin AmpErase® UNG (Applied Biosystems, California, USA), $250 \mathrm{nM}$ de cada partidor, $250 \mathrm{nM}$ de sonda y $5 \mu \mathrm{L}$ de ADN. El programa de amplificación usado en ambas RPC fue desnaturalización inicial de 20s a $95^{\circ} \mathrm{C}$, posteriormente 45 ciclos de $5 \mathrm{~s}$ a $95^{\circ} \mathrm{C}, 10 \mathrm{~s}$ a $57^{\circ} \mathrm{C}$ 
y $15 \mathrm{~s}$ a $72^{\circ} \mathrm{C}$. Los valores de CT fueron determinados automáticamente usando el software StepOne ${ }^{\mathrm{TM}}$ v2.1. Como control positivo de $B$. holmesii se utilizó una cepa previamente secuenciada para su ADNr 16S.

Secuenciación del gen recA. Para discriminar entre $B$. pertussis y $B$. holmesii se secuenció el gen recA. La amplificación del gen $\operatorname{rec} A$ fue realizada en un termociclador 2720 Thermal Cycler (Applied Biosystems) con un volumen final de $25 \mu \mathrm{L}$ que contenía tampón $1 \mathrm{x}$ (10 mM tris), $500 \mathrm{nM}$ de cada partidor (BseqF: 5'-TCCACCGGTTCGCTGGG-3' y BseqR: 5' - TGATGTCGAACTCGGCCTGC-3' Vielemeyer), 1.5 mM MgCl2, dNTPs, $1 \mathrm{U}$ taq polimerasa y $5 \mu \mathrm{L}$ de ADN. El programa de amplificación "touch-down", consta de una desnaturalización inicial de $3 \mathrm{~min}$ a $95^{\circ} \mathrm{C}$ y 10 ciclos sucesivos en los que la desnaturalización es de $30 \mathrm{~s} \mathrm{a} 95^{\circ} \mathrm{C}$, la extensión de $30 \mathrm{~s} \mathrm{a} 72^{\circ} \mathrm{C}$ y el "annealing" es de $30 \mathrm{~s}$ a $65^{\circ} \mathrm{C}$ para el primero de los 10 ciclos, con una disminución de $1^{\circ} \mathrm{C}$ en cada ciclo hasta llegar a $56^{\circ} \mathrm{C}$. A continuación hay 35 ciclos iguales de $30 \mathrm{~s}$ a $95^{\circ} \mathrm{C}, 30 \mathrm{~s}$ a $55^{\circ} \mathrm{C}$ y $30 \mathrm{~s}$ a $72^{\circ} \mathrm{C}$. La detección del producto de RPC se realizó en un gel de agarosa al 1,5\%. La RPC de secuencia tuvo un volumen final de $20 \mu \mathrm{L}$ con partidor BseqF en una concentración final $500 \mathrm{nM}$, BigDye ${ }^{\circledR}$ Terminator v3.1 Cycle Sequencing Kit y tampón 1x (Tris- $\mathrm{HCl}$, pH 9.0 and $\mathrm{MgCl}$ ). La secuenciación se realizó en el analizador genético ABI PRISM Analyzer (Applied Biosystems) y las secuencias fueron analizadas con los programas BLAST y ClustalW.

Determinación de sensibilidad analítica (Límite de detección). Para la determinación de la sensibilidad analítica se utilizaron un aislado clínico de $B$. pertussis confirmado por RPC universal con secuenciación posterior y una cepa de $B$. holmesii proveniente de la evaluación inter-laboratorios del CAP (College of American Pathologists). Ambas cepas fueron crecidas en agar Regan Lowe. Se preparó una suspensión de ambas cepas cuya concentración fue determinada por dilución y recuento en placa. Se extrajo ADN de $1 \mathrm{ml}$ de dichas suspensiones y se resuspendió en $100 \mu \mathrm{l}$ de buffer TE. Esta solución de ADN que contiene una cantidad conocida del orden de $10^{6}$ copias del genoma de Bordetella sp. por $\mu \mathrm{l}$ fue utilizada para la determinación de la sensibilidad analítica. Se prepararon diluciones seriadas 1 en 10 de estas suspensiones y se analizaron por Q-RPC.

\section{Resulltados}

Determinación de la especificidad y sensibilidad analítica de la $\boldsymbol{Q}$-RPC. Los ADN de B. pertussis y $B$. parapertussis no se detectaron con la Q-RPC específica para $B$. holmesii $(\mathrm{CT}>40)$ lo cual demuestra que es específica de esta especie.

La sensibilidad analítica de la Q-RPC dirigida a la secuencia IS481 para B. holmesii fue de 4 copias del genoma/ml de muestra, lo cual significa que el análisis es capaz de detectar $<1$ ufc/reacción. La Q-RPC dirigida al gen rec $A$ de $B$. holmesii tuvo una sensibilidad analítica de 34 copias del genoma/ml de muestra, lo cual significa que es capaz de detectar $\sim 2$ ufc/reacción (Tabla 2A). Se observa para $B$. holmesii que el CT obtenido para la amplificación de la secuencias IS481 fue alrededor de 6 ciclos menor que el CT obtenido en la amplificación del gen $r e c A$. La sensibilidad analítica de la Q-RPC dirigida a las secuencias IS481 para $B$. pertussis fue de 1 copia del genoma/ml de muestra, lo cual significa que es capaz de detectar $<1$ ufc/reacción (Tabla 2B).

Identificación de B. pertussis y B. holmesii en muestras clínicas. En el período 2010-2011 se recibieron 1.994 muestras para análisis de Bordetella sp. por Q-RPC. De las 1.994 muestras estudiadas, 224 fueron positivas para

Tabla 2. Sensibilidades analíticas de las RPC para B. holmesii (A) y para B. pertussis (B)

(A)

n de copias del genoma

B. holmesii / reacción*

17050000

1705000

170500

17050

1705

170,5

17,05

1,705

0,1705

0,01705

(B)

$\mathrm{n}$ copias del genoma

B. pertussis / reacción

$\begin{array}{lc}575000 & 12,27(11,97-12,57) \\ 57500 & 15,35(15,17-15,53) \\ 5750 & 19,89(19,34-20,43) \\ 575 & 23,39(22,65-24,13) \\ 57,5 & 27,09(26,28-27,9) \\ 5,75 & 30,88(30,37-31,29) \\ 0,575 & 33,92(33,5-34,39) \\ 0,0575 & \rightarrow 38,33(36,07-39,73) \\ 0,00575 & N D\end{array}$

ND: No determinado (sin CT). La flecha indica el límite de detección para cada blanco.

CT promedio

sonda B. pertussis IS481

sonda B. holmesii recA

$20,72(20,27-21,17)$

$23,78(23,66-23,91)$

$27,3 \quad(27,04-27,56)$

$30,77(30,49-31,05)$

$34,39(33,82-34,97)$

$37,17(36,34-38,01)$

$36,63(36,23-37,03)$

$\rightarrow 38,95(38,95-N D)$

$34,93(34,82-35,04)$

ND

---

CT promedio

sonda B. pertussis IS481

575000

57500

5750

575

\begin{tabular}{lrl}
57,5 & 27,09 & $(26,28-27,9)$ \\
5,75 & 30,88 & $(30,37-31,29)$ \\
0,575 & 33,92 & $(33,5-34,39)$ \\
0,0575 & $\rightarrow 38,33(36,07-39,73)$ \\
0,00575 & $N D$ \\
\hline
\end{tabular}


Bordetella sp. por Q-RPC dirigida a la secuencia IS481. En la Figura 1 se muestra el porcentaje de casos positivos por mes para el período del estudio. Se observa que el porcentaje de positividad fue en aumento durante el período de estudio: durante el primer semestre del año 2010 fue en promedio de $1,5 \%$, durante el segundo semestre de 2010 fue de 10,6\%, durante el primer semestre de 2011 fue de 9,9\% y durante el segundo semestre de 2011 de 14,3\%. La distribución por edades de los pacientes positivos para Bordetella sp es bimodal, tal que los casos positivos se concentran en niños bajo 1 año de edad y en adolescentes de alrededor de 14 años (Figura 2). También se observa una mayor incidencia en niños bajo 6 meses de edad que en niños entre 7-12 meses.

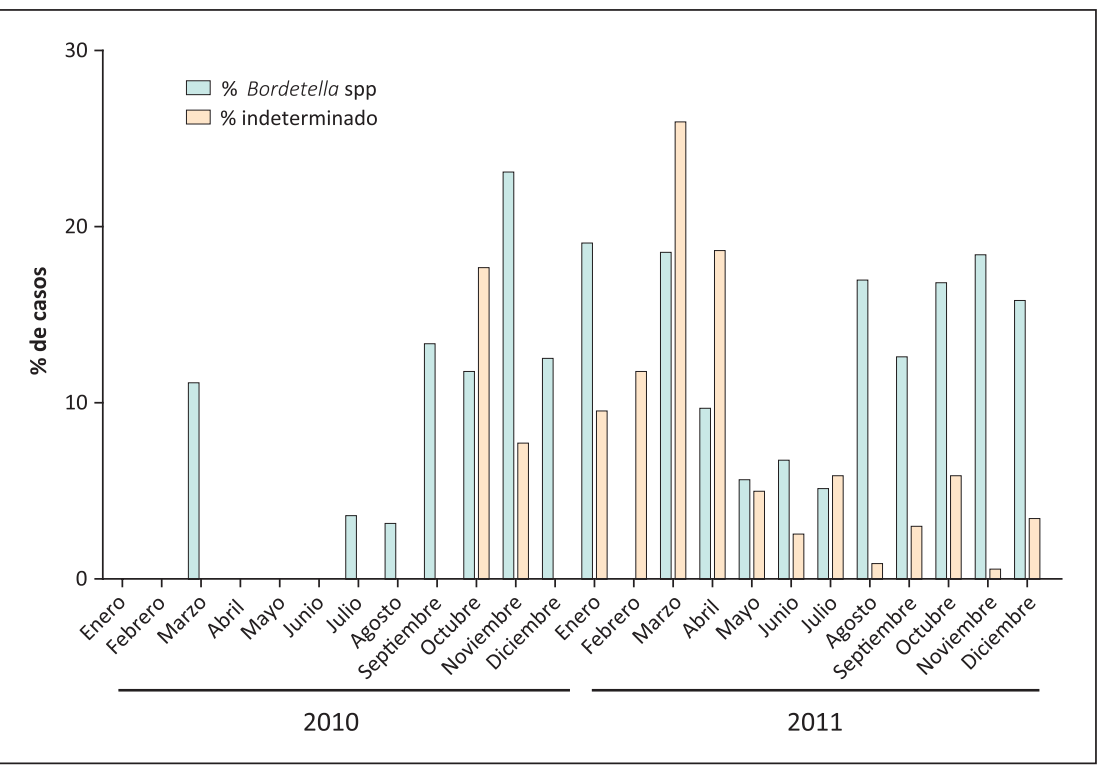

Figura 1. Porcentaje de casos de Bordetella spp y de indeterminados para el período estudiado. Se expresa el porcentaje del total de muestras recibidas para análisis de Bordetella. Los casos de Bordetella spp son todos aquellos que dieron positivo para la secuencia IS481 e incluyen por lo tanto B. pertussis, B. holmesii y $B$. bronchiseptica.

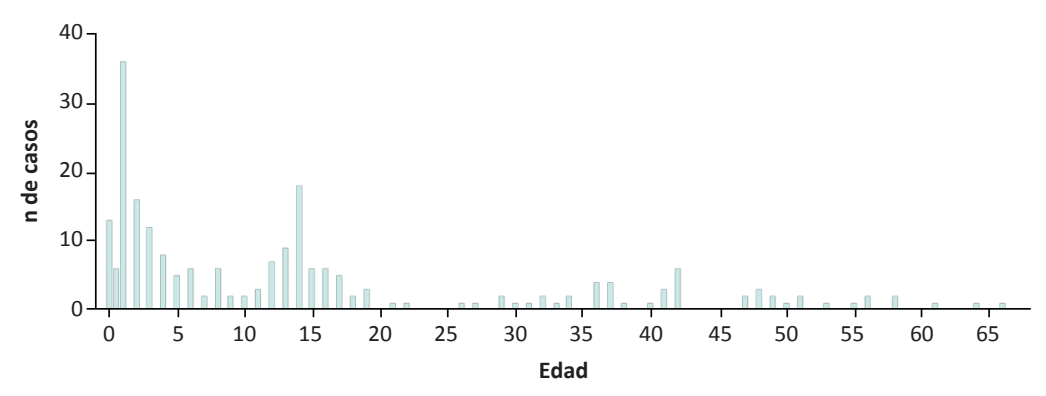

Figura 2. Distribución etaria de los casos positivos de Bordetella (positivos para RPC en tiempo real dirigido a la secuencia IS481). Las edades están expresadas en años excepto en las dos primeras barras del gráfico que corresponden a 0-6 meses y 7-12 meses.
Desde el segundo semestre del año 2010 comenzaron a detectarse muestras indeterminadas con valores de CT entre 35 y 40. Durante el segundo semestre del año 2010 estas muestras representaron $3,5 \%$, en el primer semestre del 2011 el 7,9\% y en el segundo semestre de 2011 el 3,2 $\%$ (Figura 1). Se observa en la Figura 1 que a medida que aumentan los casos de Bordetella sp, también aumentan los casos indeterminados. Respecto de la secuenciación del gen recA para determinar si eran $B$. pertussis o $B$. holmesii, no fue posible amplificar ADN por RPC convencional en ninguna muestra indeterminada debido a la baja carga bacteriana encontrada en esas muestras. Se secuenció entonces el gen rec $A$ de 6 muestras positivas (CT menor a 35) de este período elegidas al azar. La secuenciación demostró que 5 de ellas correspondían a $B$. pertussis y una a $B$. holmesii, lo cual confirmó la existencia de esta especie en Chile. La Figura 3 muestra el alineamiento del gen rec $A$ de $B$. holmesii, B. pertussis y del aislado clínico de $B$. holmesii secuenciado en nuestro laboratorio. Luego de este hallazgo se analizaron, retrospectiva y prospectivamente, todas las muestras positivas del período 2010-2011 mediante Q-RPC específica para B. holmesii. De las 224 muestras positivas para Bordetella sp., 12 fueron positivas para B. holmesii (5.4\%) lo cual da una frecuencia de esta especie en el período de $0,6 \%$. La Figura 4 muestra el número de casos de $B$. holmesii y $B$. pertussis para cada mes del período estudiado. Se observa que el número de casos de $B$. pertussis aumenta notoriamente a lo largo del período y que la mayoría de los casos de $B$. holmesii se presentó en el primer semestre de 2011, durante el período de brote de B. pertussis.

\section{Discusión}

En este trabajo se reporta por primera vez la presencia de $B$. holmesii en Chile, encontrando el primer caso en una muestra respiratoria recibida en agosto del año 2010. La presencia de $B$. holmesii, especialmente durante brotes de coqueluche, también ha sido recientemente reportada en otros países como por ejemplo en Ohio, E.U.A. ${ }^{20}$. Además, la frecuencia de $B$. holmesii encontrada en la Red de Salud UC concuerda con la encontrada en otras regiones. De acuerdo a trabajos previos, la prevalencia de $B$. holmesii en general está alrededor de $1 \%{ }^{15,11,21}$. En un estudio realizado en Massachusetts, E.U.A., durante el año 1998 se encontró una prevalencia de $B$. holmesii de $0,6 \%$ del total de muestras recibidas para análisis de Bordetella sp. En otro estudio en Ontario, Canadá, entre 2007 y 2008 se encontró una prevalencia menor a 1\% entre todas las muestras recibidas para análisis de Bordetella sp. ${ }^{11}$. Sin embargo, durante una evaluación retrospectiva de la presencia de $B$. holmesii en Finlandia entre los años 2000 al 2003 y en Países Bajos desde 1992 al 2003, no se encontró la presencia del patógeno ${ }^{21}$. Recientemente fue 
publicado un trabajo por nuestro grupo donde se reporta una prevalencia de $B$. holmesii de $11,1 \%{ }^{16}$. La diferencia entre el $11,1 \%$ descrito en dicho trabajo y el $5,4 \%$ en el presente trabajo se debe a que en el presente artículo fueron agregadas las muestras del segundo semestre de 2011 entre las cuales hubo sólo un caso de B. holmesii y, por lo tanto, disminuyó el porcentaje global de la misma.

Los valores de sensibilidad analítica obtenidos en este trabajo son comparables a los obtenidos por Tatti y cols. ${ }^{22}$. La diferencia de entre 5 y 6 ciclos entre el CT obtenido para la secuencia IS481 y para el gen $r e c A$ de $B$. holmesii se explica por el mayor número de copias en que se encuentra IS481 con respecto al gen recA. Los valores de sensibilidad analítica son menores cuanto mayor es el número de copias del gen blanco. El número de copias de IS481 en $B$. pertussis es de 80-100, en $B$. holmesii es de 8-10 y el de rec $A$ en $B$. holmesii es de 1, y concordantemente tienen sensibilidades analíticas de $0,06,0,2$ y 1,7 , respectivamente.

Se han evaluado otras estrategias moleculares en busca de darle mayor especificidad a la RPC en tiempo real. Así, expertos europeos recomiendan trabajar simultáneamente con los partidores para IS481 y para el gen $p t x$ (codifica el pertusinógeno); con la primera amplificación se obtiene buena sensibilidad y la segunda agrega especificidad a la $\mathrm{RPC}^{23}$. Lo mismo han concluido expertos canadienses ${ }^{24}$. Otros autores han recomendado ensayar una amplificación multiplex incluyendo IS481, IS1001 e IS1002 para distinguir las diferentes especies de Bordetella ${ }^{25}$. Sería ventajoso poder en un futuro realizar la detección del gen del pertusinógeno ya que si bien su presencia no asegura que sea $B$. pertussis, entregaría información acerca de la virulencia de la cepa que está infectando el paciente. Dada la metodología descrita en este artículo, no es posible determinar si hay co-infección. No fue posible diferenciar si las muestras indeterminadas corresponden a $B$. holmesii o $B$. pertussis. La RPC convencional es menos sensible que la Q-RPC y dado que para secuenciar es necesario una cantidad considerable de producto de RPC convencional, no fue posible secuenciar las muestras que tienen $\mathrm{CT}$ entre 35 y 40 . Estas muestras podrían corresponder a pacientes que estaban enfermos y recibieron una terapia antimicrobiana que disminuyó drásticamente la carga bacteriana. Los CT entre 35 y 40 podrían deberse también a que los síntomas se ven atenuados por la vacunación y por ende a una baja carga de microorganismo. En un trabajo realizado durante un brote de $B$. pertussis en Canadá $^{26}$, la mayoría de los casos tenían CT elevados (alrededor de 39) y una muestra fue considerada positiva cuando tenía un $\mathrm{CT} \leq 40$ ciclos. Sin embargo muchos de los pacientes con exámenes positivos no presentaban los síntomas típicos. En ausencia de síntomas clásicos, una RPC positiva para $B$. pertussis puede representar una forma atípica de la enfermedad o colonización transitoria.

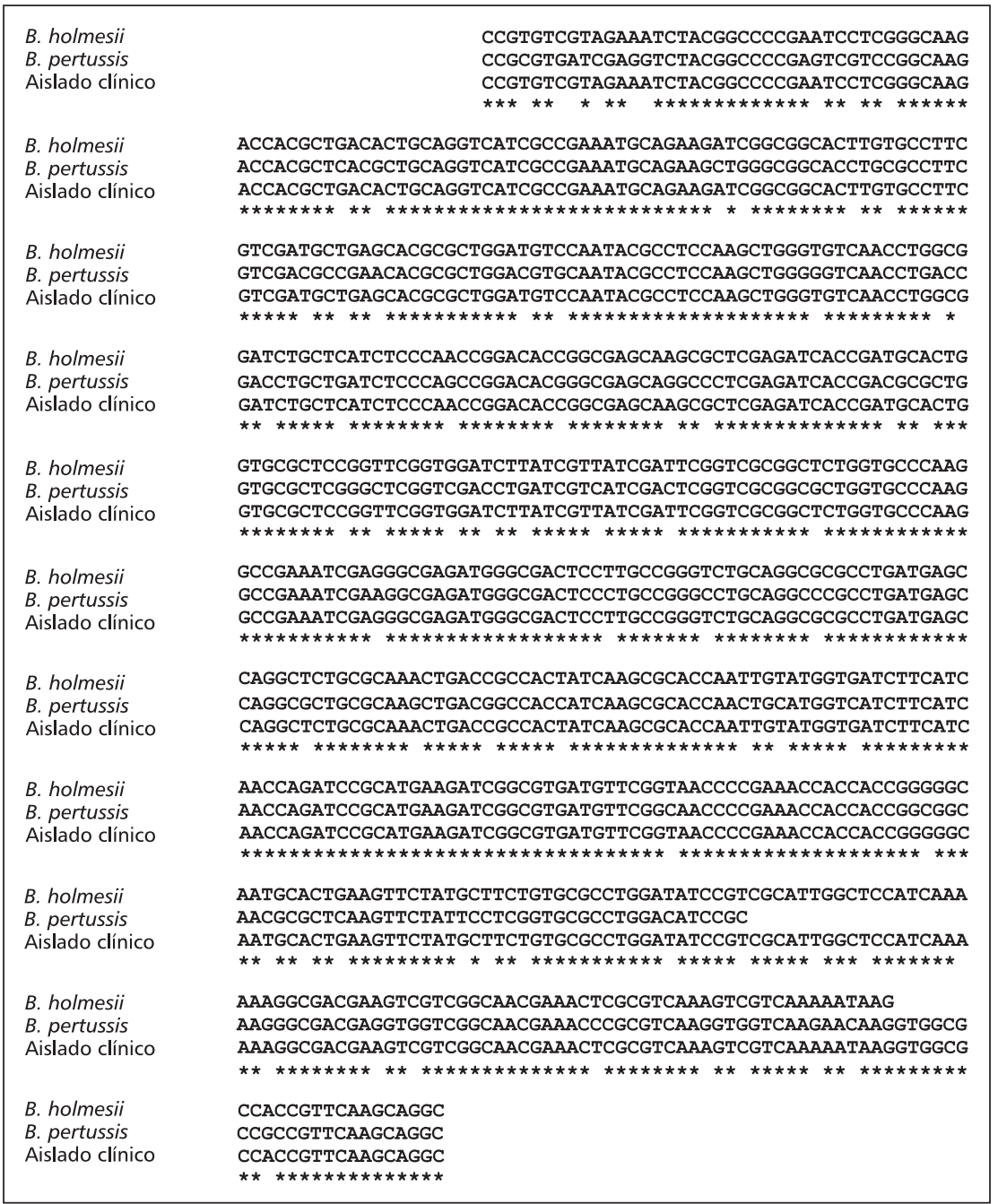

Figura 3. Alineamiento de la secuencia parcial del gen recA de $B$. holmesii ( $N^{\circ}$ acceso de Genbank AF399661.2), de B. pertussis (AF399658.1) y del aislado clínico que resultó ser B. holmesii. El alineamiento fue realizado con el programa ClustalW. Los asteriscos se encuentran en las posiciones en las cuales las tres secuencias tienen la misma base. Las posiciones en las cuales no hay asterisco corresponden a diferencias entre $B$. pertussis y $B$. holmesii.

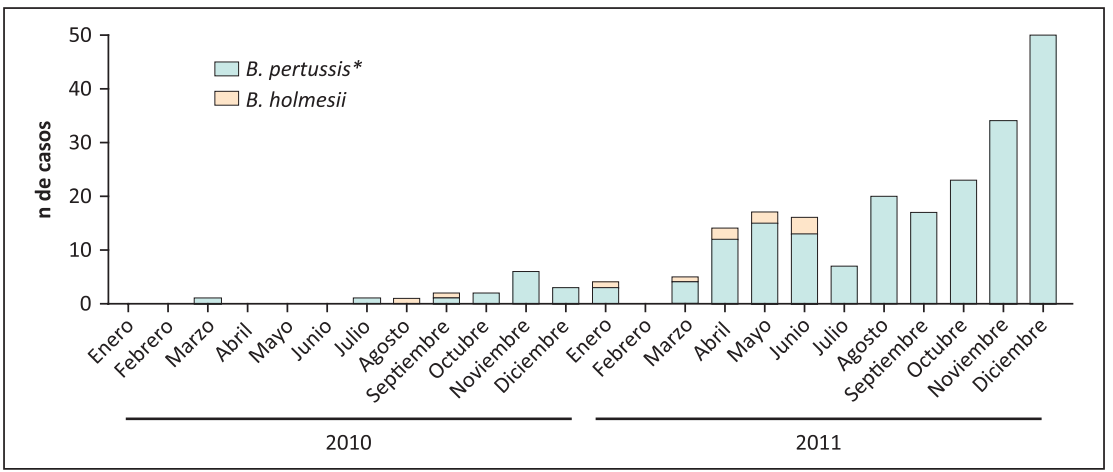

Figura 4. $\mathrm{N}^{\circ}$ de casos de $B$. pertussis y de B. holmesii para el período estudiado. * Los casos de $B$. pertussis son todos aquellos que dieron positivo para la secuencia IS481 y negativo para la RPC dirigida al gen recA de $B$. holmesii. 
De acuerdo a estos antecedentes, en nuestro trabajo las muestras que tuvieron resultados con CT entre 35 y 40 y que fueron informados como indeterminados podrían ser consideradas como portadores del microorganismo. Guthrie $^{6}$ describe que aquellas muestras que tengan un CT $>35$, corresponden a muestras que tienen $<1$ ufc de Bordetella sp en la muestra utilizada para hacer RPC. Estos pacientes podrían ser portadores del microorganismo y no cumplir con la definición de caso clínico de la OMS. Varios estudios han demostrado que puede darse la portación de $B$. pertussis ${ }^{27,28}$ en especial en períodos de brotes. Para conocer si estas muestras corresponden a pacientes con clínica concordante al cuadro respiratorio se debe evaluar conjuntamente con los antecedentes clínicos del paciente. Por lo tanto, la interpretación de los valores de CT $>35$ debería hacerse en el contexto del caso clínico particular. Es esperable que en situaciones de brote aumente la portación del patógeno porque el aumento de casos de $B$. pertussis favorecería la aparición de portadores con baja carga bacteriana. Esto es lo que pudo haber ocurrido a partir del segundo semestre de 2010 en Chile, momento en que se produjo un brote de este patógeno y un aumento del número de casos con baja carga bacteriana. El brote ocurrido en nuestro país también fue evidenciado en este estudio, donde se describe un aumento importante en el número de casos positivos para Bordetella sp a partir del segundo semestre de 2010. También es interesante la distribución etaria de los casos positivos observada en este trabajo, probablemente explicada por la estrategia de vacunación nacional, resultando en un aumento de casos en niños bajo 6 meses de edad correspondiente a la población susceptible pre-vacunación y en el grupo de 14 años de edad, en quienes la inmunidad conferida por la vacuna comienza a decaer ${ }^{29}$.

A raíz de este hallazgo y dada la importancia de diferenciar entre ambas especies de Bordetella, en nuestro laboratorio se decidió realizar la identificación de $B$. pertussis y $B$. holmesii en todas las muestras que llegan para análisis de Bordetella sp. Esta determinación adicional no afecta mayormente los costos porque se realiza solamente en las muestras positivas para Bordetella sp
(IS481) que en período de brote corresponde a $11,2 \%$ de las muestras recibidas y en período sin brote a $6 \%$. Con respecto al tiempo de respuesta, los casos positivos se informan como positivos para Bordetella sp con lo cual el médico está informado que se trata de Bordetella. Sin embargo, la determinación de especie pertussis u holmesii se realiza 24 h después.

Agradecimientos. Agradecemos al personal del laboratorio de Microbiología de la PUC por su colaboración en la realización de este trabajo. Agradecemos al Programa SENTRY por prover fondos, que fueron destinados a la realización de este trabajo.

\section{Resumen}

La incidencia de coqueluche en Chile varía entre 4,1 y 7,5 por 100.000 habitantes. La detección de Bordetella pertussis se realiza por RPC-tiempo real (Q-RPC) dirigida a la secuencia de inserción IS481. Sin embargo, esta secuencia se encuentra también en el genoma de $B$. bronchiseptica y $B$. holmesii. Este último es también un patógeno respiratorio que produce un cuadro similar a $B$. pertussis. Sin embargo, es importante diferenciar entre estas especies porque en pacientes inmunosuprimidos $B$. holmesii tiene mayor tendencia a causar bacteriemia y además es menos susceptible a eritromicina. El objetivo de este trabajo es determinar, prospectiva y retrospectivamente, la presencia de $B$. holmesii en muestras informadas positivas para $B$. pertussis en el período 2010-2011. Durante ese período ingresaron al laboratorio 1.994 muestras de hisopado nasofaríngeo para RPC de Bordetella sp., de las cuales 224 fueron positivas. El análisis por Q-RPC dirigido al gen recA de $B$. holmesii de las 224 muestras positivas determinó una prevalencia de $B$. holmesii de $0,6 \%$ (12/1994). Debido al comportamiento más agresivo en inmunosuprimidos y al patrón de resistencia de $B$. holmesi, se decide incorporar la detección de rutina de $B$. pertussis y $B$. holmesii en todas las muestras en que se detecta inicialmente la presencia de Bordetella $\mathrm{sp}$.

\section{Referencias bibliográficas}

1.- Mattoo S, Cherry J D. Molecular pathogenesis, epidemiology, and clinical manifestations of respiratory infections due to Bordetella pertussis and other Bordetella subspecies. Clin Microbiol Rev 2005; 18 (2): 326-82.

2.- Gobierno de Chile. Ministerio de Salud. Circular $N^{\circ}$ B51/04: Vigilancia epidemiológica y medidas de control de coqueluche (tos ferina). 2007. http://www.epi.minsal.cl

3.- Gobierno de Chile. Ministerio de Salud.
Circular $\mathrm{N}^{\circ}$ B51/27: Vigilancia epidemiológica y medidas de control de coqueluche (tos ferina) 2011. http://www.epi.minsal.cl

4.- Summaries of Infectious Diseases. Pertussis (Whooping Cough). En Red Book 2012, (ed. K. P. Larry), pp. 553-566: American Academy of Pediatrics.

5.- Guiso N, Wirsing von Konig C H, Forsyth K, Tan T, Plotkin S A. The Global Pertussis Initiative: report from a round table meeting to discuss the epidemiology and detection of pertussis, Paris, France, 11-12 January 2010.
Vaccine 2011; 29 (6): 1115-21.

6.- Guthrie J L, Seah C, Brown S, Tang P, Jamieson F, Drews S J. Use of Bordetella pertussis BP3385 to establish a cutoff value for an IS481-targeted real-time PCR assay. J Clin Microbiol 2008; 46 (11): 3798-9.

7.- Furuya D, Yagihashi A, Endoh T, Uehara N, Fujii N, Chiba S, et al. Simultaneous amplification of Bordetella repeated insertion sequences and toxin promoter region gene by polymerase chain reaction. Immunopharmacol Immunotoxicol 1999; 21 (1): 55-63. 
8.- Kosters K, Riffelmann M, Wirsing von Konig $\mathrm{C}$ H. Evaluation of a real-time PCR assay for detection of Bordetella pertussis and $B$. parapertussis in clinical samples. J Med Microbiol 2001; 50 (5): 436-40.

9.- Register K B, Sanden G N. Prevalence and sequence variants of IS481 in Bordetella bronchiseptica: implications for IS481-based detection of Bordetella pertussis. J Clin Microbiol 2006; 44 (12): 4577-83.

10.- Reischl U, Lehn N, Sanden GN, Loeffelholz M J. Real-time PCR assay targeting IS481 of Bordetella pertussis and molecular basis for detecting Bordetella holmesii. J Clin Microbiol 2001; 39 (5): 1963-6.

11.- Guthrie J L, Robertson A V, Tang P, Jamieson F, Drews S J. Novel duplex real-time PCR assay detects Bordetella holmesii in specimens from patients with Pertussis-like symptoms in Ontario, Canada. J Clin Microbiol 2010; 48 (4): 1435-7.

12.- Weyant R S, Hollis D G, Weaver R E, Amin M F, Steigerwalt AG, O'Connor S P, et al. Bordetella holmesii sp. nov., a new gram-negative species associated with septicemia. J Clin Microbiol 1995; 33 (1): 1-7.

13.- Mazengia E, Silva E A, Peppe J A, Timperi R, George H. Recovery of Bordetella holmesii from patients with pertussis-like symptoms: use of pulsed-field gel electrophoresis to characterize circulating strains. J Clin Microbiol 2000; 38 (6): 2330-3.

14.- Tang Y W, Hopkins M K, Kolbert C P, Hartley P A, Severance P, Persing D H. Bordetella holmesii-like organisms associated with septicemia, endocarditis, and respiratory failure. Clin Infect Dis 1998; 26 (2): 389-92.
15.- Yih W K, Silva E A, Ida J, Harrington N, Lett SM, George H. Bordetella holmesii-like organisms isolated from Massachusetts patients with pertussis-like symptoms. Emerg Infect Dis 1999; 5 (3): 441-3.

16.- Miranda C, Porte L, García P. Bordetella holmesii in nasopharyngeal samples from Chilean patients with suspected Bordetella pertussis infection. J Clin Microbiol 2012; 50, 1505; author reply 1506 .

17.- Woolfrey B, Moody J. Human infections associated with Bordetella bronchiseptica. Clin Microbiol Rev 1991; 4: 243-255.

18.- Shepard C W, Daneshvar M I, Kaiser R M, Ashford D A, Lonsway D, Patel J B et al. Bordetella holmesii bacteremia: a newly recognized clinical entity among asplenic patients. Clin Infect Dis 2004; 38 (6): 799-804.

19.- Gobierno de Chile. Ministerio de Salud. Informe de coqueluche año 2011. http://www.epi.minsal. cl. Octubre 2011. http://epi.minsal.cl/epi/html/ bolets/reportes/Coqueluche/Tos_Final_2011.pdf.

20.- Rodgers L, Martin S W, Cohn A, Budd J, Marcon M, Terranella A, et al. Epidemiologic and laboratory features of a large outbreak of pertussis-like illnesses associated with cocirculating Bordetella holmesii and Bordetella pertussis - Ohio, 2010-2011. Clin Infect Dis. 2013. Feb; 56 (3): 322-31. doi: 10.1093/cid/ cis888. Epub 2012 Oct 19.

21.- Antila M, He Q, de Jong C, Aarts I, Verbakel H, Bruisten $\mathrm{S}$ et al. Bordetella holmesii DNA is not detected in nasopharyngeal swabs from Finnish and Dutch patients with suspected pertussis. J Med Microbiol 2006; 55 (Pt 8): 1043-51.

22.- Tatti K M, Sparks K N, Boney K O, Tondella M L. Novel multitarget real-time
PCR assay for rapid detection of Bordetella species in clinical specimens. J Clin Microbiol 2011; 49 (12): 4059-66.

23.- Meade B D, Bollen A. Recommendations for use of the polymerase chain reaction in the diagnosis of Bordetella pertussis infections. J Med Microbiol 1994; 41: 51-5.

24.- Proceedings of the National Microbiology Laboratory Pertussis Workshop. Winnipeg, Manitoba, 7 March, 2006. Canada Communicable Disease Report 2006 (Nov): Vol 32 S4.

25.- Roorda L, Buitenwerf J, Ossewaarde J, Anneke van der Zee. A real-time PCR assay with improved specificity for detection and discrimination of all clinically relevant Bordetella species by the presence and distribution of three insertion sequence elements. BMC Research Notes 2011; 4: 11

26.- Waters V, Jamieson F, Richardson S E, Finkelstein M, Wormsbecker A, Halperin S A. Outbreak of atypical pertussis detected by polymerase chain reaction in immunized preschool-aged children. Pediatr Infect Dis J 2009; 28 (7): 582-7.

27.- Klement E, Uliel L, Engel I, Hasin T, Yavzori M, Orr N, et al. An outbreak of pertussis among young Israeli soldiers. Epidemiol Infect 2003; 131 (3): 1049-54.

28.- Srugo I, Benilevi D, Madeb R, Shapiro S, Shohat T, Somekh E, et al. Pertussis infection in fully vaccinated children in day-care centers, Israel. Emerg Infect Dis 2000; 6 (5): 526-9.

29.- Wendelboe A, Van Rie A, Salmaso S, Englund J. Duration of immunity against pertussis after natural infection or vaccination. Pediatr Infect Dis J 2005; 24: S58-61. 